Case Report

\title{
Severe Rhabdomyolysis as Complication of Interaction between Atorvastatin and Fusidic Acid in a Patient in Lifelong Antibiotic Prophylaxis: A Dangerous Combination
}

\author{
Anirban Nandy ${ }^{1}$ and Shahin Gaïni ${ }^{1,2,3}$ \\ ${ }^{1}$ Medical Department, Infectious Diseases Division, National Hospital Faroe Islands, Tórshavn, Faroe Islands \\ ${ }^{2}$ Infectious Diseases Research Unit, Odense University Hospital and University of Southern Denmark, Odense, Denmark \\ ${ }^{3}$ Faculty of Science and Technology, University of the Faroe Islands, Tórshavn, Faroe Islands \\ Correspondence should be addressed to Anirban Nandy; anirban_198798@yahoo.co.in
}

Received 28 September 2016; Revised 13 November 2016; Accepted 4 December 2016

Academic Editor: Mamede de Carvalho

Copyright (C) 2016 A. Nandy and S. Gaïni. This is an open access article distributed under the Creative Commons Attribution License, which permits unrestricted use, distribution, and reproduction in any medium, provided the original work is properly cited.

\begin{abstract}
Atorvastatin and HMG-CoA reductase inhibitors are the most frequently used medication in the world due to very few adverse toxic side effects. One potentially life threatening adverse effect is caused by clinically significant statin induced rhabdomyolysis, either independently or in combination with fusidic acid. The patient in our case who previously had cardiac insufficiency, atrial fibrillation, and thoracic aorta aneurysm and was treated with insertion of an endovascular metallic stent in the aorta is presented in the report. He had an inoperable aortitis with an infected stent and para-aortic abscesses with no identified microorganism. The patient responded well to empirical antibiotic treatment with combination therapy of fusidic acid and moxifloxacin. This treatment was planned as a lifelong prophylactic treatment. The patient had been treated with atorvastatin for several years. He developed severe rhabdomyolysis when he was started on fusidic acid and moxifloxacin. The patient made a fast recovery after termination of treatment with atorvastatin and fusidic acid. We here report a life threatening complication of rhabdomyolysis that physicians must be aware of. This can happen either in atorvastatin monotherapy or as a complication of pharmacokinetic interaction between atorvastatin and fusidic acid.
\end{abstract}

\section{Introduction}

Rhabdomyolysis is most commonly described by "lysis" or disintegration and breakdown of skeletal muscles and subsequent release of toxic intracellular components into the systemic circulation. The major causes of rhabdomyolysis include trauma, infections, hyperthermia, myopathies, and adverse drug-drug interactions of certain medications [1]. Statin myotoxicity is a well-known side effect and is related to serum levels of the drugs and can also be influenced by coprescription with other drugs, thus increasing the risk of side effects including rhabdomyolysis [2-4]. Although it has been reported previously that there is an increased risk of myopathy with coprescription of atorvastatin and fusidic acid, it was not before 2011 that the avoidance of coprescription of this drug combination was recommended [5-7]. We present a patient already on statin treatment who developed rhabdomyolysis apparently precipitated by fusidic acid. The potential life threatening interaction between atorvastatin and fusidic acid is highlighted in this case report.

\section{Case Presentation}

A Caucasian man aged 75 years presented to the Emergency Department at the National Hospital, Faroe Islands, with complaints of a two-week history of progressively severe diffuse myalgia, confined to the lower back and proximal lower muscles, reduced power in all four extremities, and generalized weakness, and he was immobile and bedridden. He was neurologically sound and there was no complaint regarding headache, numbness, tingling, or paraesthesia. $\mathrm{He}$ denied indulgence in any kind of vigorous physical exercise 
TABLE 1: Muscle and kidney function biomarkers during the admission. The combination of atorvastatin and fusidic acid stopped at day 6 since admission.

\begin{tabular}{|c|c|c|c|c|}
\hline $\begin{array}{l}\text { Days } \\
\text { (according to admission) }\end{array}$ & $\begin{array}{c}\text { Creatinine kinase } \\
\text { (normal 40-280 U/L) }\end{array}$ & $\begin{array}{c}\text { Myoglobulin } \\
\text { (normal 24-77 ng/L) } \\
\end{array}$ & $\begin{array}{c}\text { Creatinine } \\
\text { (normal 60-105 micromole/L) }\end{array}$ & $\begin{array}{c}\text { Urea } \\
\text { (normal 3.5-8.1 mmol/L) }\end{array}$ \\
\hline 1 & - & - & 128 & 16.8 \\
\hline 2 & - & - & - & - \\
\hline 3 & - & - & - & - \\
\hline 4 & - & - & 119 & - \\
\hline 5 & 10865 & - & 108 & - \\
\hline 6 & 10750 & - & 92 & - \\
\hline 7 & 22520 & - & 134 & - \\
\hline 8 & 35080 & - & 134 & - \\
\hline 9 & $>20000$ & 26300 & 100 & 22.2 \\
\hline 10 & 8070 & 7110 & 85 & 21.8 \\
\hline 11 & 3350 & 2590 & 74 & 19.5 \\
\hline 12 & 1740 & - & 74 & 17.0 \\
\hline 13 & 755 & - & 62 & 13.3 \\
\hline 14 & 314 & - & 70 & 10.4 \\
\hline 15 & 197 & 143 & 68 & 8.1 \\
\hline 16 & 149 & 89 & 81 & 7.7 \\
\hline 17 & 112 & 94 & 79 & 8.0 \\
\hline 22 & 389 & - & 81 & 8.0 \\
\hline 26 & 72 & - & 85 & - \\
\hline 29 & 71 & - & 85 & - \\
\hline
\end{tabular}

and over consumption of alcohol. He was already under medication for hypertension and in treatment with rivaroxaban due to atrial fibrillation and atorvastatin $80 \mathrm{mg}$ daily for hyperlipidaemia and on lifelong prophylactic empirical antibiotic treatment with oral fusidic acid $500 \mathrm{mg}$ twice a day combined with oral moxifloxacin $400 \mathrm{mg}$ daily and for an infected aortic aneurysm, with an infected endovascular aortic stent and para-aortic abscesses. He had no familial or prior personal history of muscle disorder and no past history of muscular toxicity with statin or fibrate use. He had been in treatment with atorvastatin for several years. He had been treated with fusidic acid and moxifloxacin for 6 days before the day of admission.

He was afebrile with heart rate 75 beats/minute but hypotensive (103/42 $\mathrm{mmHg}$ ). The cardiovascular, respiratory, and gastrointestinal tract examination was normal. He complained of moderate discomfort due to myalgia. The quadriceps muscles were tender to palpation and he had reduced power bilaterally. The cranial nerves 2-12 examination was normal. The power $5 / 5$ strength in all the muscle groups was observed, except for the hip flexors and quadriceps which rated $3 / 5$ bilaterally. He was immobile due to weakness. He had normal sensory examinations to light touch, pin prick, and proprioception.

On admission the laboratory measurements revealed the following: hemoglobin $6.4 \mathrm{mmol} / \mathrm{L}$, total leukocyte count $7.8 \times 10^{9} / \mathrm{L}$, potassium $4.4 \mathrm{mmol} / \mathrm{L}$, sodium $140 \mathrm{mmol} / \mathrm{L}$, urea $16.8 \mathrm{mmol} / \mathrm{L}$, creatinine $128 \mathrm{micromol} / \mathrm{L}$, and blood glucose $6.5 \mathrm{mmol} / \mathrm{L}$. Serum muscle enzymes were markedly elevated: creatinine kinase $(\mathrm{CK}) 328 \mathrm{U} / \mathrm{L}$ (reference values: 40-208), LDH $266 \mathrm{U} / \mathrm{L}$ (reference values: 115-255), BNP $607 \mathrm{pmol} / \mathrm{L}$ (normal up to 28.9), elevated liver function test with ALAT $125 \mathrm{U} / \mathrm{L}$ (reference values: 10-70), alkaline phosphatases $232 \mathrm{U} / \mathrm{L}$ (reference values: 35-105), and normal bilirubin $9 \mathrm{U} / \mathrm{L}$ (reference values: 5-25). Table 1 describes the levels of CK, myoglobin, creatinine, and urea (the combination was stopped on 6th day after admission). Urine analysis showed no significant microscopic hematuria or proteinuria. The patient was also screened for autoimmune diseases: autoantibody p-ANCA (IgG) with medium positive result (nonconclusive), autoantibody c-ANCA (IgG) negative, and autoantibody ANA negative. These results made the differential diagnoses immunological vasculitis, granulomatosis with polyangiitis, and systemic lupus erythematosus (SLE) unlikely. The rheumatological differential diagnoses polymyositis and dermatomyositis were unlikely because of negative autoantibodies known to be associated to polymyositis and dermatomyositis (Table 2). A spinal tap was performed to rule out Guillain-Barré syndrome as differential diagnosis. The spinal tap showed no pleocytosis, normal protein, and normal glucose level in the cerebrospinal fluid. An MRI of the cervical spine showed a nonsignificant spinal stenosis at the C5/C6 level, which did not explain the symptomatology of the patient. Finally because of negative HMG-CoA reductase antibodies (IgG), associated to autoimmune statin induced rhabdomyolysis, this differential diagnosis was unlikely in our patient (Table 2). Therefore, considering the clinical development, the kinetics of the 
TABLE 2: Screening for autoantibodies known to be associated to polymyositis, dermatomyositis, and isolated statin (HMG-CoA reductase inhibitor) causing myalgia.

\begin{tabular}{lc}
\hline Antibodies & Results \\
\hline P-Glycyl-tRNA synthetase-Ab. (IgG) & Negative \\
P-Jo 1-antibody (IgG) & Negative \\
P-Histidine-tRNA-ligase (Jo1)-Ab. (IgG) & Negative \\
P-Isoleucyl-tRNA synth. cytop.-Ab. (IgG) & Negative \\
P-MDA5-antibody (IgG) & Negative \\
P-Mi-2a-antibody (IgG) & Negative \\
P-NXP2-antibody (IgG) & Negative \\
P-Polymyositis (Ku)-A (IgG) & Negative \\
P-Polymyositis (PL-12)-antibody & Negative \\
P-Polymyositis (PL-7)-Ab. (IgG) & Negative \\
P-Polymyositis (SRP)-Ab. (IgG) & Negative \\
P-SAE1-antibody (IgG) & Negative \\
P-TIF1 y-antibody (IgG) & Negative \\
HMG-CoA reductase-Ab. (IgG) (HMGCR), & $<3$ \\
normal reference < 20 & \\
\hline
\end{tabular}

biochemical markers, and the medication history, we assume that the diagnosis of rhabdomyolysis as a consequence to combination therapy with atorvastatin and fusidic acid is highly likely. Treatment with atorvastatin and fusidic acid was halted on 6th day after admission and approximately 12 th day since the combination started. Fusidic acid was changed to oral Co-amoxiclav 500/125 mg tid and the patient continued on atorvastatin and oral moxifloxacin $400 \mathrm{mg}$ daily. The patient was treated with intravenous fluid and physiotherapy.

The patient was discharged after 5 weeks with remarkable improvement of symptoms. When he left hospital the biochemical markers revealed normal muscle enzymes with CK $71 \mathrm{U} / \mathrm{L}$, myoglobin $94 \mathrm{ng} / \mathrm{L}$, and normal transaminases (Table 1 and Figure 1). It was decided not to treat the patient with fusidic acid in the future.

\section{Discussion}

Statins are HMG-CoA inhibitors (hydroxymethylglutaryl/ coenzyme A) and they lower the cholesterol levels by competitively inhibiting the HMG-CoA reductase, the final pathway in cholesterol biosynthesis $[4,8]$. The three statins (lovastatin, simvastatin, and atorvastatin) are metabolized by the cytochrome p-450 3A4 (CYP-3A4) isoenzymes, whereas pravastatin is metabolized in the liver by sulfation and fluvastatin is metabolized by the cytochrome 2C9 isoenzyme (CYP-2C9) [8].

Rhabdomyolysis is one of the complications of myopathies. The risk of myopathies and rhabdomyolysis is increased by the concomitant administration of fusidic acid with statins [4]. Plasma concentration of statins significantly increases by coadministration of fusidic acid and HMG-CoA reductase inhibitor $[4,7,9]$. The mechanism of interaction, whether it is pharmacodynamics or pharmacokinetics or both, is still unknown. Reports have shown rhabdomyolysis,

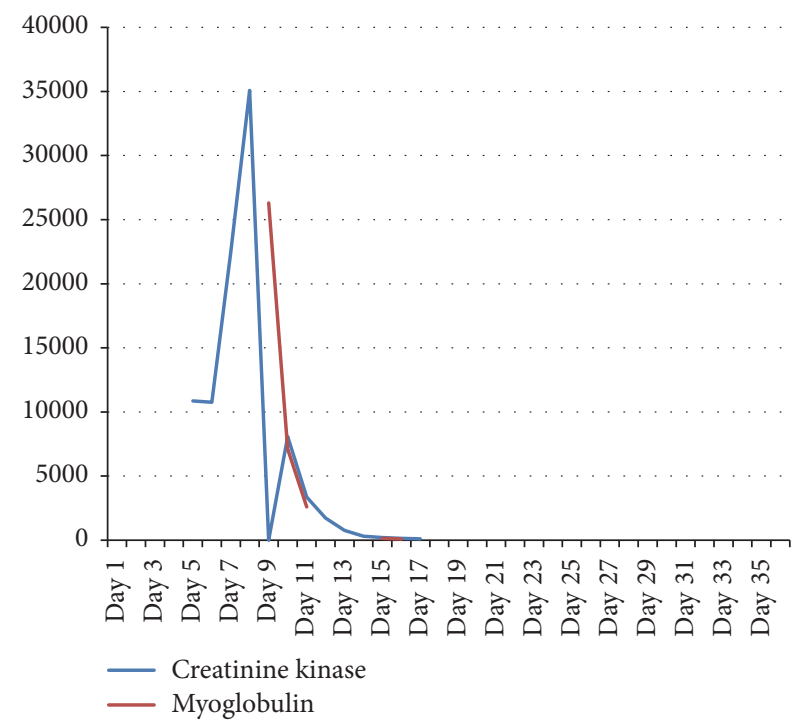

FIGURE 1: The graph shows the levels of creatinine kinase (CK) and myoglobulin in the $y$-axis and the admission days in the $x$-axis. The rapid fall and normalization of the CK and myoglobulin in our patient, after cessation of treatment with atorvastatin and fusidic acid on 6th day prior to admission, clearly suggest the diagnosis of pharmacokinetic interaction between atorvastatin and fusidic acid as cause of rhabdomyolysis.

including some fatal cases, in patients receiving combination of fusidic acid and its salts with oral anticoagulants such as warfarin, other coumarin derivatives, or anticoagulant; this may also increase plasma concentration of these anticoagulant agents, thus enhancing the anticoagulant effects with risk of bleeding. An interaction between fusidic acid and drugs biotransformed via CYP-3A4 system is suspected. Apparently the mechanism of this interaction is possibly mutual inhibition of metabolism [9]. However, recent studies suggest fusidic acid inhibits hepatic transporters and metabolic enzymes which may cause drug-drug interaction with statin coadministration [10].

The mortality rate associated with statin induced rhabdomyolysis is approximately 0.15 deaths per 1 million [4, 11] and it is considered as a rare side effect involving less than $0.1 \%$ of patients on statin treatment. The FDA database reports a mortality rate of $7.8 \%$ in patients with rhabdomyolysis $[4,12]$. Cerivastatin was previously one of the most commonly implicated statin $[4,11]$. Due to more than 100 fatal outcomes linked to rhabdomyolysis, it was withdrawn from the market in August $2001[4,12]$.

Factors increasing the plasma concentration of the statins increase the risk of rhabdomyolysis and hepatitis. These include concomitant use of lipid lowering drugs, hosts genetic factors, and drug interactions with other medications that are metabolized by the same cytochrome $\mathrm{p}-450$ system of enzymes [7, 13]. Risk factors for these adverse effects include renal disease, hepatic dysfunction, diabetes, age above 80 years, and hypothyroidism $[4,14,15]$. The most common medications affecting statin metabolism, apart from fusidic 
acid, are fibrates (especially gemfibrozil), cyclosporine, warfarin, digoxin, macrolides, azole antifungals, calcium channel blockers, and amiodarone [2, 14-16].

The mainstay treatment of rhabdomyolysis is hydration and increasing diuresis. Diuresis protects the kidneys by diluting myoglobin in the renal tubules and hence prevents the toxic cast formation and also promotes the excretion [17]. Mannitol and urine alkalinization are also indicated in the treatment of rhabdomyolysis. To our knowledge there are no specific guidelines, but The American Academy of Clinical Toxicology and the European Association of Poisons Centre and Clinical Toxicologists have issued a paper recommending administration of a bolus of $225 \mathrm{~mL}$ of $8.4 \%$ sodium bicarbonate intravenously over $1 \mathrm{~h}$, followed by additional intravenous boluses q. $1 \mathrm{~h}$, to maintain $\mathrm{pH}$ between 7.5 and 8.5. Another regimen is to add $1-3 \mathrm{amps}(50 \mathrm{mEq} / 50 \mathrm{cc})$ of sodium bicarbonate from $8.4 \%$ to $0.9 \%$ or $0.45 \%$ normal saline or 5\% D5W [18]. In all regimes, high recommendations have been made to monitor the $\mathrm{pH}$, serum potassium, and arterial pH hourly $[7,19]$.

\section{Conclusion}

This case report is highlighting the importance of early recognition and treatment of this rare but potentially fatal side effect, rhabdomyolysis, caused by use of the statin group of drugs, either separately or combined with fusidic acid. If statins are used to treat patients, fusidic acid should not be used. In conditions where life threatening infection is involved and fusidic acid is the only antibiotic option available, it is suggested that all statin treatments should be set on a halt, as long as the patient is receiving fusidic acid.

\section{Consent}

Written consent for a case report publication was obtained from the patient in April 2016.

\section{Competing Interests}

The authors declare that there is no conflict of interests regarding the publication of this article.

\section{Authors' Contributions}

Anirban Nandy is involved in patient follow-up and wrote the manuscript. Shahin Gaïni treated and diagnosed the patient and contributed to the manuscript draft.

\section{References}

[1] D. J. Graham, J. A. Staffa, D. Shatin et al., "Incidence of hospitalized rhabdomyolysis in patients treated with lipid-lowering drugs," The Journal of the American Medical Association, vol. 292, no. 21, pp. 2585-2590, 2004.

[2] M. A. Omar, J. P. Wilson, and T. S. Cox, "Rhabdomyolysis and HMG-CoA reductase inhibitors," Annals of Pharmacotherapy, vol. 35, no. 9, pp. 1096-1107, 2001.
[3] C. M. Ballantyne, A. Corsini, M. H. Davidson et al., "Risk for myopathy with statin therapy in high-risk patients," Archives of Internal Medicine, vol. 163, no. 5, pp. 553-564, 2003.

[4] P. D. Thompson, P. Clarkson, and R. H. Karas, "Statin-associated myopathy," JAMA, vol. 289, no. 13, pp. 1681-1690, 2003.

[5] A. J. Burtenshaw, G. Sellors, and R. Downing, "Presumed interaction of fusidic acid with simvastatin," Anaesthesia, vol. 63, pp. 656-658, 2008.

[6] C. Magee, S. A. Medani, S. F. Leavey, P. J. Conlon, and M. R. Clarkson, "Severe rhabdomyolysis as a consequence of the interaction of fusidic acid and atorvastatin," American Journal of Kidney Diseases, vol. 56, pp. el1-e15, 2010.

[7] D. H. Schreiber and T. R. Anderson, "Statin-induced rhabdomyolysis," Journal of Emergency Medicine, vol. 31, no. 2, pp. 177-180, 2006.

[8] R. H. Knopp, "Drug treatment of lipid disorders," The New England Journal of Medicine, vol. 341, no. 7, pp. 498-511, 1999.

[9] C. Wenisch, R. Krause, P. Fladerer, I. El Menjawi, and E. Pohanka, "Acute rhabdomyolysis after atorvastatin and fusidic acid therapy," American Journal of Medicine, vol. 109, article no. 78, 2000.

[10] A. Gupta, J. J. Harris, J. Lin, J. P. Bulgarelli, B. K. Birmingham, and S. W. Grimm, "Fusidic acid inhibits hepatic transporters and metabolic enzymes: potential cause of clinical drug-drug interaction observed with statin coadministration," Antimicrobial Agents and Chemotherapy, vol. 60, no. 12, pp. 5986-5994, 2016.

[11] P. H. Chong, J. D. Seeger, and C. Franklin, "Clinically relevant differences between the statins: implications for therapeutic selection," American Journal of Medicine, vol. 111, no. 5, pp. 390400, 2001.

[12] V. Fuhrmans, "Bayer discloses higher death toll from Baycol," Wall Street Journal, vol. A10, 2002.

[13] J. Martin and H. Krum, "Cytochrome p450 drug interactions within the HMG-CoA reductase inhibitor class: are they clinically relevant?” Drug Safety, vol. 26, no. 1, pp. 13-21, 2003.

[14] J. P. Deslypere and A. Vermeulen, "Rhabdomyolysis and simvastatin," Annals of Internal Medicine, vol. 114, no. 4, p. 342, 1991.

[15] R. J. Herman, "Drug interactions and the statins," CMAJ, vol. 161, no. 10, pp. 1281-1286, 1999.

[16] D. Williams and J. Feely, "Pharmacokinetic-pharmacodynamic drug interactions with HMG-CoA reductase inhibitors," Clinical Pharmacokinetics, vol. 41, no. 5, pp. 343-370, 2002.

[17] L. J. Bontempo, "Rhabdomyolysis," in Rosen's Emergency Medicine, J. Marx and R. Walls, Eds., pp. 1762-1769, Mosby, New York, NY, USA, 5th edition, 2002.

[18] R. Vanholder, M. S. Sever, E. Erek, and N. Lameire, "Rhabdomyolysis," Journal of the American Society of Nephrology, vol. 11, no. 8, pp. 1553-1561, 2000.

[19] C. E. Haas, Y. Magram, A. Mishra, L. González, and J.-M. Kaiser, "Rhabdomyolysis and acute renal failure following an ethanol and diphenhydramine overdose," Annals of Pharmacotherapy, vol. 37, no. 4, pp. 538-542, 2003. 


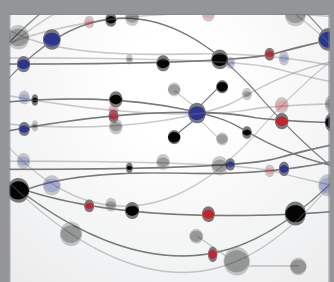

The Scientific World Journal
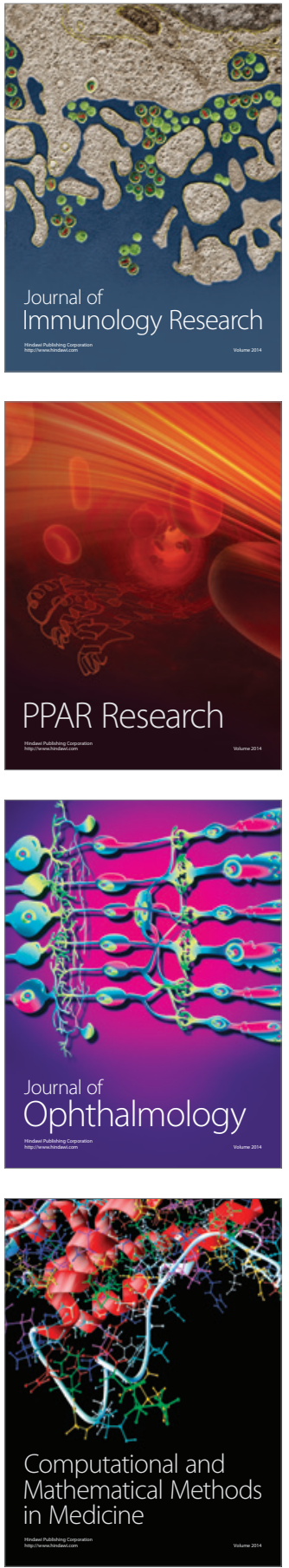

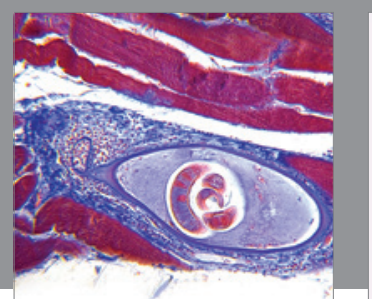

Gastroenterology Research and Practice

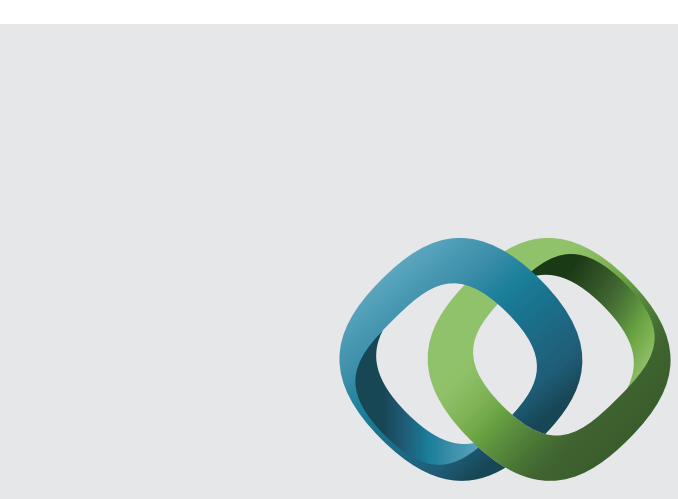

\section{Hindawi}

Submit your manuscripts at

http://www.hindawi.com
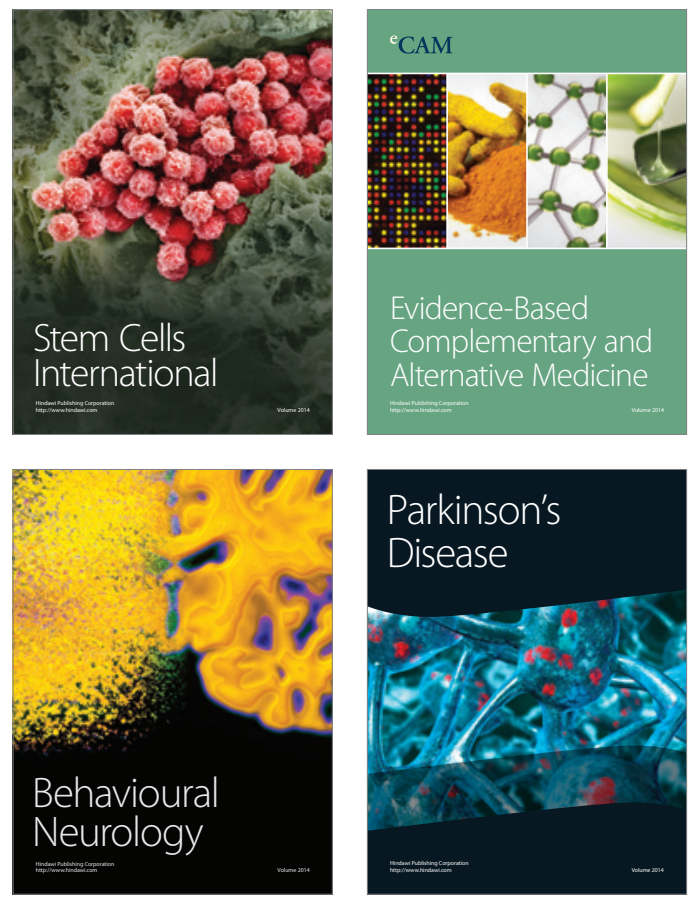
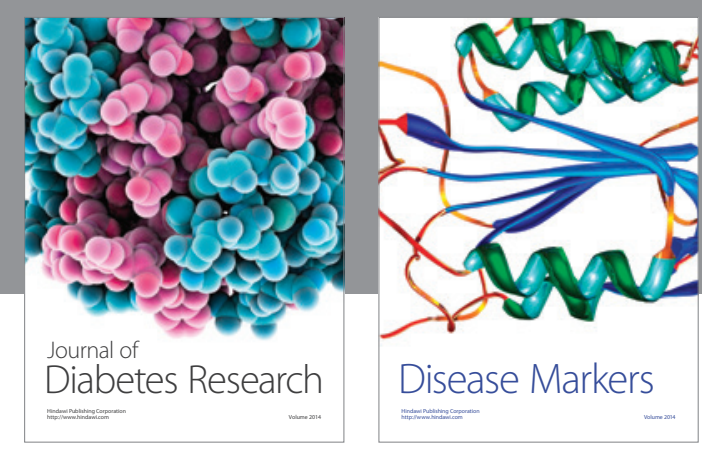

Disease Markers
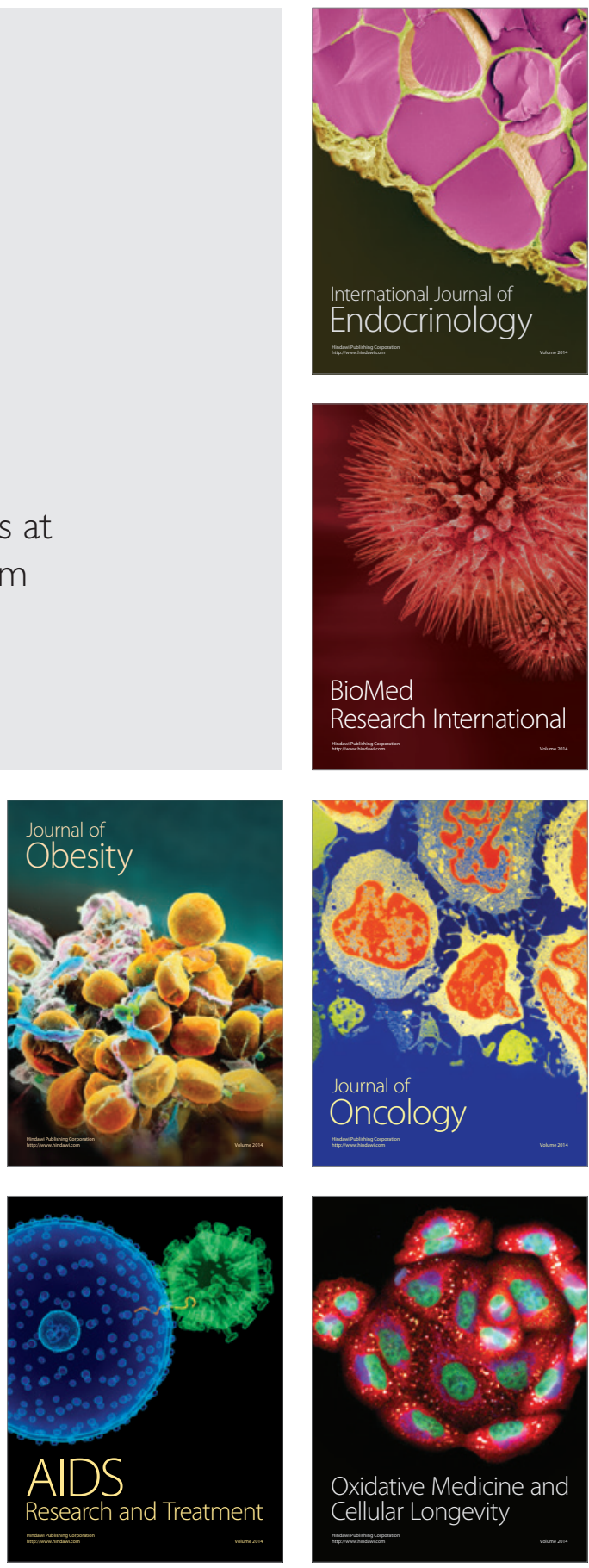